\title{
FORMULAS FOR LINEAR SUMS THAT INVOLVE GENERALIZED FIBONACCI AND LUCAS NUMBERS
}

\author{
ZVONKO ČERIN
}

\begin{abstract}
We extend Melham's formulas in [16] for certain classes of finite sums that involve generalized Fibonacci and Lucas numbers. We study the linear sums where only single terms of these numbers appear. Our results show that most of his formulas are the initial terms of a series of formulas, that the analogous and somewhat simpler identities hold for associated dual numbers and that besides the alternation according to the numbers $(-1)^{\frac{n(n+1)}{2}}$ it is possible to get similar formulas for the alternation according to the numbers $(-1)^{\frac{n(n-1)}{2}}$. We also consider sixteen linear sums with binomial coefficients that are products.
\end{abstract}

\section{INTRODUCTION}

The main goal of this paper and its sequel [5] is to improve several results by R. S. Melham in [16]. His idea was to consider identities for those finite sums of (products of at most two) generalized Fibonacci and Lucas numbers where the right hand side has a pleasant form. In order to achieve a balance between elegance and generality, he choose to employ the following four sequences that we now define.

Let $a, b$ and $p$ be arbitrary complex numbers such that $p \neq 0$ and $p^{2} \neq$ $-2,-4$. The roots $\alpha=\frac{p+\sqrt{\Delta}}{2}$ and $\beta=\frac{p-\sqrt{\Delta}}{2}$, where $\Delta=p^{2}+4$, of the equation $z^{2}-p z-1=0$ are distinct (because $p^{2} \neq-4 \Leftrightarrow \Delta \neq 0$ ). The values $p=0$ and $p^{2}=-2$ are excluded because they give sequences that are not interesting. The first two sequences are given by their Binet forms as

$$
W_{n}=\frac{A \alpha^{n}-B \beta^{n}}{\alpha-\beta} \quad \text { and } \quad X_{n}=A \alpha^{n}+B \beta^{n}
$$

for any integer $n$, where $A=b-a \beta$ and $B=b-a \alpha$.

For $(a, b)=(0,1)$, we write $W_{n}=U_{n}$ and $X_{n}=V_{n}$. Then $\left\{U_{n}\right\}_{n \in \mathbb{Z}}$ and $\left\{V_{n}\right\}_{n \in \mathbb{Z}}$ are the third and the fourth sequences, respectively.

2010 Mathematics Subject Classification. Primary: 11B39, 11Y55, 05A19.

Key words and phrases. (Generalized) Fibonacci number, (generalized) Lucas number, factor, sum, alternating, binomial coefficient, product.

Copyright (c) 2015 by ANUBiH. 
Of course, these numbers can also be defined recursively since the identities $W_{0}=a, W_{1}=b, W_{n}=p W_{n-1}+W_{n-2}$ and $X_{0}=2 b-a p, X_{1}=2 a+$ $b p, X_{n}=p X_{n-1}+X_{n-2}$ are true for all integers $n$. Moreover, $W_{n}$ and $X_{n}$ generalize $U_{n}$ and $V_{n}$, respectively, which in turn generalize $F_{n}$ and $L_{n}$ (Fibonacci and Lucas numbers), respectively. Aspects of $W_{n}$ and $X_{n}$ have been treated, for example, in [1], [13], and [21], and more recently in [15].

We shall employ also another four similar sequences that we call dual and denote by $w_{n}, x_{n}, u_{n}$ and $v_{n}$ (the corresponding small letters) which come from the equation $z^{2}-p z+1=0$ under the assumption that $p \neq 0$ and $p^{2} \neq 2,4$. Let $\delta=p^{2}-4$. For example, the numbers $w_{n}$ satisfy $w_{0}=a$, $w_{1}=b, w_{n}=p w_{n-1}-w_{n-2}$ (for $\left.n \geq 2\right)$ and are given explicitly by $w_{n}=$ $\frac{C \varphi^{n}-D \psi^{n}}{\varphi-\psi}$, where $\varphi=\frac{p+\sqrt{\delta}}{2}, \psi=\frac{p-\sqrt{\delta}}{2}, C=b-a \psi$ and $D=b-a \varphi$.

Aspects of $u_{n}$ and $v_{n}$ have been treated in [9] and [11]. These papers mention that the sequence $\left\{u_{n}\right\}$ generates the natural numbers when $p=2$ and that for $p>2$, some familiar properties of the natural numbers can be found in $\left\{u_{n}\right\}$ (for example, the formula $\sum_{i=1}^{n} u_{2 i-1}=u_{n}^{2}$ is analogous to the familiar identity $\left.\sum_{i=1}^{n}(2 i-1)=n^{2}\right)$. Also, for $p=3$, we have $u_{n}=F_{2 n}$ and $v_{n}=L_{2 n}$ while for $p=6$, we have $u_{n}=P_{2 n}$ (the Pell subsequence).

As in [16], in each of our sums the lower limit is allowed to vary. Accordingly, we always assume the upper limit to be greater than the lower limit, and that either limit may be negative.

In Sections 2-9 we present our results that are collected into eight sets of sums covering the linear sums in Sections 2 and 3 of [16] and then conclude by giving in Section 10 a sample proof.

\section{THE FIRST SET OF SUMS}

In the formula (2.1) in [16] (see also [3] and [4]), Melham proved that the sums of either $4 k+1$ or $4 k+3$ consecutive terms of the sequence $\left(W_{j}\right)_{j \in \mathbb{Z}}$ is a simple product. Our first two formulas show that the same holds for all sequences $\left(W_{s j}\right)_{j \in \mathbb{Z}}$, where $s$ is any integer. Hence, instead of a single formula we obtain a whole sequence of formulas (one for each value of $s$ ).

Let $i, k$ and $s$ denote arbitrary integers. Define linear functions $\mathfrak{E}, \mathfrak{O}: \mathbb{Z} \rightarrow$ $\mathbb{Z}$ by $\mathfrak{E}(s)=\underline{s}=2 s$ and $\mathfrak{O}(s)=\bar{s}=2 s+1$ for every $s \in \mathbb{Z}$. In order to get short formulas we shall use these two functions $\mathfrak{E}$ (for even) and $\mathfrak{O}$ (for odd) (i. e., letters with underline and/or overline) and the following five substitutions (i. e., short names) $\ell=k+i, m=k-i, \lambda=2 k+i, \mu=2 k+i+1$ and $\nu=2 k+i+2$.

$$
V_{\bar{s}} \sum_{j=i}^{i+n} W_{\bar{s} j}= \begin{cases}V_{\bar{s} \bar{k}}\left[W_{\bar{s} \lambda}+W_{\bar{s} \mu}\right], & \text { if } n=4 k+1 \\ U_{\bar{s}(\bar{k}+1)}\left[X_{\bar{s} \mu}+X_{\bar{s} \nu}\right], & \text { if } n=4 k+3\end{cases}
$$


The next two series of identities cover the case when instead of the odd multiples $\bar{s} j$ of the index $j$ we consider its even multiples $\underline{s} j$.

$$
U_{\underline{s}} \sum_{j=i}^{i+n} W_{\underline{s} j}= \begin{cases}\left.U_{\underline{s}} \bar{k}\left[W_{\underline{s} \lambda}+W_{\underline{s}}\right]\right], & \text { if } n=4 k+1 ; \\ U_{\underline{s}(\bar{k}+1)}\left[W_{\underline{s}}+W_{\underline{s}}\right], & \text { if } n=4 k+3 .\end{cases}
$$

These sums for the numbers $w_{n}$ are always products (regardless of how many terms are in the sum) and have the following forms that depend only on the parity of the number of terms.

$$
u_{s} \sum_{j=i}^{i+n} w_{s j}= \begin{cases}{\left[u_{s(k+1)}+u_{s k}\right] w_{s \ell},} & \text { if } n=\underline{k} \\ u_{s(k+1)}\left[w_{s(\ell+1)}+w_{s \ell}\right], & \text { if } n=\bar{k}\end{cases}
$$

\section{The SECOnd SET OF Sums}

The following identities give an analogous extension of the formula (2.2) in $[16]$.

$$
\begin{aligned}
& V_{\bar{s}} \sum_{j=i}^{i+n}(-1)^{i+j} W_{\bar{s} j}= \begin{cases}V_{\bar{s} \bar{k}}\left[W_{\bar{s} \lambda}-W_{\bar{s} \mu}\right], & \text { if } n=4 k+1 ; \\
U_{\bar{s}(\bar{k}+1)}\left[X_{\bar{s} \mu}-X_{\bar{s} \nu}\right], & \text { if } n=4 k+3 .\end{cases} \\
& U_{\underline{s}} \sum_{j=i}^{i+n}(-1)^{i+j} W_{\underline{s} j}= \begin{cases}U_{\underline{s} \bar{k}}\left[W_{\underline{s} \lambda}-W_{\underline{s}} \mu\right], & \text { if } n=4 k+1 \\
U_{\underline{s}(\bar{k}+1)}\left[W_{\underline{s} \mu}-W_{\underline{s} \nu}\right], & \text { if } n=4 k+3 .\end{cases}
\end{aligned}
$$

These sums for the numbers $w_{n}$ are again always products (regardless of how many terms are in the sum).

$$
\begin{aligned}
& u_{\bar{s}} \sum_{j=i}^{i+n}(-1)^{i+j} w_{\bar{s} j}= \begin{cases}{\left[u_{\bar{s}(k+1)}-u_{\bar{s} k}\right] w_{\bar{s} \ell},} & \text { if } n=\underline{k} ; \\
u_{\bar{s}(k+1)}\left[w_{\bar{s} \ell}-w_{\bar{s}(\ell+1)}\right], & \text { if } n=\bar{k} .\end{cases} \\
& v_{s} \sum_{j=i}^{i+n}(-1)^{i+j} w_{\underline{s} j}= \begin{cases}v_{s \bar{k}} w_{\underline{s}} \ell, & \text { if } n=k \\
-u_{\underline{s}(k+1)} x_{\underline{s} \ell+s}, & \text { if } n=\bar{k} .\end{cases}
\end{aligned}
$$

\section{THE THIRD SET OF SUMS}

The extension of the identity (2.3) in [16] consists of the following group of formulas. Let $M_{1}$ and $M_{2}$ denote $\left[U_{\bar{s}(k+1)}+(-1)^{i} U_{\bar{s} k}\right] W_{\bar{s} \ell}$ and $U_{\bar{s}(k+1)}$ $\left[W_{\bar{s}(\ell+1)}-(-1)^{i} W_{\bar{s} \ell}\right]$, respectively.

$$
(-1)^{k} U_{\bar{s}} \sum_{j=i}^{i+n}(-1)^{\frac{j(j+1)}{2}} W_{\bar{s} j}= \begin{cases}(-1)^{\frac{i(i+1)}{2}} M_{1}, & \text { if } n=\underline{k} \\ (-1)^{\frac{(i+1)(i+2)}{2}} M_{2}, & \text { if } n=\bar{k}\end{cases}
$$


Let $M_{3}$ and $M_{4}$ stand for the products $V_{\underline{s} \bar{k}}\left[(-1)^{i} W_{\underline{s} \lambda}-W_{\underline{s}} \mu\right]$ and $U_{\underline{s}}(\bar{k}+1)$ $\left[X_{\underline{s} \nu}-(-1)^{i} X_{\underline{s} \mu}\right]$, respectively.

$$
(-1)^{\frac{i(i-1)}{2}} V_{\underline{s}} \sum_{j=i}^{i+n}(-1)^{\frac{j(j+1)}{2}} W_{\underline{s} j}= \begin{cases}M_{3}, & \text { if } n=4 k+1 ; \\ M_{4}, & \text { if } n=4 k+3 .\end{cases}
$$

Here it is possible to consider the same sums using the alternation according to $(-1)^{\frac{j(j-1)}{2}}$ instead of the one according to $(-1)^{\frac{j(j+1)}{2}}$. The formulas for these sums differ only slightly from the formula for the original sum (usually in signs of two terms). For example, the corresponding identity for the first half of (4.1) above is the following formula (note the change of $(-1)^{\frac{i(i+1)}{2}}$ into $(-1)^{\frac{i(i-1)}{2}}$ and of the plus sign into the minus sign in the bracket). Let $M_{1}^{*}$ be $\left[U_{\bar{s}(k+1)}-(-1)^{i} U_{\bar{s} k}\right] W_{\bar{s} \ell}$.

$$
(-1)^{k} U_{\bar{s}} \sum_{j=i}^{i+\underline{k}}(-1)^{\frac{j(j-1)}{2}} W_{\bar{s} j}=(-1)^{\frac{i(i-1)}{2}} M_{1}^{*} .
$$

For the dual numbers $w_{n}$ these sums are somewhat simpler again. Let $m_{3}=v_{s \bar{k}}\left[(-1)^{i} w_{s \lambda}-w_{s \mu}\right]$ and $m_{4}=u_{s(\bar{k}+1)}\left[x_{s \nu}+(-1)^{i} x_{s \mu}\right]$.

$$
(-1)^{\frac{i(i-1)}{2}} v_{s} \sum_{j=i}^{i+n}(-1)^{\frac{j(j+1)}{2}} w_{s j}= \begin{cases}m_{3}, & \text { if } n=4 k+1 ; \\ m_{4}, & \text { if } n=4 k+3 .\end{cases}
$$

The alternation according to $(-1)^{\frac{j(j-1)}{2}}$ in the first part of (4.4) is given by the following identity. Similar changes occur in the second part of (4.4).

$$
(-1)^{\frac{i(i+1)}{2}} v_{s} \sum_{j=i}^{i+4 k+1}(-1)^{\frac{j(j-1)}{2}} w_{s j}=v_{s \bar{k}}\left[(-1)^{i} w_{s \lambda}+w_{s \mu}\right] .
$$

It is interesting that in the above sums we can add different terms and still end with almost the same formula. For example, for the first part of (4.1) this modification gives the following identity.

$$
U_{\bar{s}} \sum_{j=0}^{\underline{k}}(-1)^{\frac{j(j+1)}{2}} W_{i+\bar{s} j}=(-1)^{k}\left[U_{\bar{s}(k+1)}+U_{\bar{s} k}\right] W_{i+\bar{s} k} .
$$

\section{The Fourth SeT OF SUMS}

The formula (3.1) in [16] is also the first of a whole series of formulas.

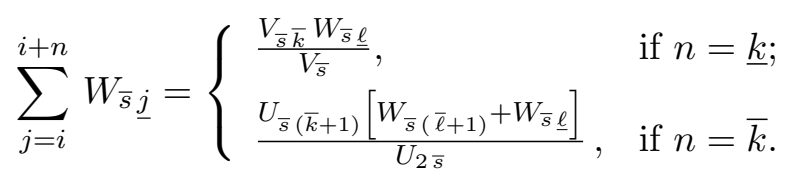




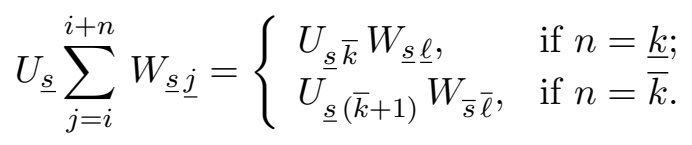

Another way to write the first part of (5.2) is the following.

$$
U_{\underline{\underline{s}}} \sum_{j=i}^{i+\underline{k}} W_{\underline{s} \underline{j}}=\left[U_{\underline{s}(\bar{k}+1)}+U_{\underline{s} \underline{k}}\right] W_{\underline{s} \underline{\ell}} .
$$

For the numbers $w_{n}$ these formulas are as follows. We simply replace big letters with small and only in the third formula $V$ is twice replaced with $u$ and not with $v$. They also follow from formula (2.3) for $s=4 s+2$ and $s=4 s$. The identity $u_{s}\left[w_{\underline{s}(k+1)}+w_{\underline{s} k}\right]=u_{\underline{s}} w_{s \bar{k}}$ and its special case when $w$ is replaced with $u$ imply the following shorter forms.

$$
u_{s} \sum_{j=i}^{i+\underline{k}} w_{\underline{s} j}=u_{s \bar{k}} w_{\underline{s} \ell}, \quad u_{\underline{s}} \sum_{j=i}^{i+\bar{k}} w_{\underline{s} j}=u_{\underline{s}(\bar{k}+1)} w_{\underline{s} \bar{\ell}} .
$$

The formula (3.3) in [16] can be extended in a similar way.

$$
\begin{gathered}
\sum_{j=i}^{i+n}(-1)^{i+j} W_{\bar{s} \underline{j}}= \begin{cases}\frac{U_{\bar{s} \bar{k}} W_{\bar{s} \underline{\ell}}}{U_{\bar{s}}}, & \text { if } n=\underline{k} ; \\
\frac{U_{\bar{s}(\bar{k}+1)}\left[W_{\bar{s} \underline{\ell}}-W_{\bar{s}(\bar{\ell}+1)}\right]}{U_{2 \bar{s}}}, & \text { if } n=\bar{k} .\end{cases} \\
V_{\underline{s}} \sum_{j=i}^{k+n}(-1)^{i+j} W_{\underline{s} \underline{j}}= \begin{cases}V_{\underline{s} \bar{k}} W_{\underline{s} \underline{\ell}}, & \text { if } n=\underline{k} ; \\
-U_{\underline{s}(\bar{k}+1)} X_{\underline{s} \bar{\ell}}, & \text { if } n=\bar{k} .\end{cases}
\end{gathered}
$$

The above remark applies again to the versions of these formulas for the numbers $w_{n}$. This time, in the first part of (5.6), the letter $U$ is twice replaced with $v$ and not with $u$. Of course, these formulas are much simpler because there is no need to consider separately even and odd multiples of $\underline{j}$.

$$
v_{s} \sum_{j=i}^{i+n}(-1)^{i+j} w_{s \underline{j}}= \begin{cases}v_{s \bar{k}} x_{\underline{s}} \ell, & \text { if } n=\underline{k} ; \\ -u_{\underline{s}(k+1)} x_{s \bar{\ell}}, & \text { if } n=\bar{k} .\end{cases}
$$

\section{The FIFTH SET OF SUMS}

The following is the extension of the formula (3.4) in [16]. Recall that $m$ is the difference $k-i$ and $\ell$ is the sum $k+i$.

$$
\frac{V_{2 z}}{U_{4 z m}} \sum_{j=4 i+1}^{4 k}(-1)^{\frac{j(j+1)}{2}} W_{z} \underline{j}= \begin{cases}\Delta U_{z} W_{z(4 \ell+1)}, & \text { if } z=\bar{s} \\ V_{z} X_{z(4 \ell+1)}, & \text { if } z=\underline{s} .\end{cases}
$$


Once more, these sums for the numbers $w_{n}$ are simpler.

$$
v_{\underline{s}} \sum_{j=4 i+1}^{4 k}(-1)^{\frac{j(j+1)}{2}} w_{s \underline{j}}=v_{s} u_{\underline{s} \underline{m}} x_{s(4 \ell+1)} \text {. }
$$

The following two formulas cover the alternation according to the numbers $(-1)^{\frac{j(j-1)}{2}}$ in (6.1) and (6.2).

$$
\begin{gathered}
\frac{V_{2 z}}{U_{4 z m}} \sum_{j=4 i+1}^{4 k}(-1)^{\frac{j(j-1)}{2}} W_{z \underline{j}}= \begin{cases}V_{z} X_{z(4 \ell+1)}, & \text { if } z=\bar{s} \\
\Delta U_{z} W_{z(4 \ell+1)}, & \text { if } z=\underline{s} .\end{cases} \\
v_{\underline{s}} \sum_{j=4 i+1}^{4 k}(-1)^{\frac{j(j-1)}{2}} w_{s \underline{j}}=\delta u_{s} u_{\underline{s} \underline{m}} w_{s(4 \ell+1) .}
\end{gathered}
$$

The following is the extension of the formula (3.5) in [16].

$$
\frac{V_{2 z}}{V_{2 z(2 m-1)}} \sum_{j=4 i+3}^{4 k}(-1)^{\frac{j(j+1)}{2}} W_{z} \underline{j}= \begin{cases}U_{z} X_{z(4 \ell+3)}, & \text { if } z=\bar{s} \\ V_{z} W_{z(4 \ell+3)}, & \text { if } z=\underline{s} .\end{cases}
$$

This sum for the numbers $w_{n}$ is the following.

$$
v_{\underline{s}} \sum_{j=4 i+3}^{4 k}(-1)^{\frac{j(j+1)}{2}} w_{s \underline{j}}=v_{s} v_{\underline{s}(2 m-1)} w_{s(4 \ell+3)} .
$$

The following identity extends the formula (3.6) in [16].

$$
\frac{V_{2 z}}{V_{4 z(m+1)}} \sum_{j=4 i}^{4 k+3}(-1)^{\frac{j(j+1)}{2}} W_{z \underline{j}}= \begin{cases}U_{z} X_{z(4 \ell+3)}, & \text { if } z=\bar{s} \\ \Delta U_{z} W_{z(4 \ell+3)}, & \text { if } z=\underline{s} .\end{cases}
$$

Once again for the numbers $w_{n}$ this sum is simpler.

$$
v_{\underline{s}} \sum_{j=4 i}^{4 k+3}(-1)^{\frac{j(j+1)}{2}} w_{s \underline{j}}=\delta u_{s} u_{4 \underline{s}(m+1)} w_{s(4 \ell+3)} .
$$

The following identity improves the formula (3.7) in [16].

$$
\frac{V_{2 z}}{V_{2 z \bar{m}}} \sum_{j=4 i+2}^{4 k+3}(-1)^{\frac{j(j+1)}{2}} W_{z \underline{j}}= \begin{cases}V_{z} W_{\bar{s}(4 \ell+5),} & \text { if } z=\bar{s} \\ U_{z} X_{\underline{s}}(4 \ell+5), & \text { if } z=\underline{s} .\end{cases}
$$

Here is the corresponding identity for the numbers $w_{n}$.

$$
v_{\underline{s}} \sum_{j=4 i+2}^{4 k+3}(-1)^{\frac{j(j+1)}{2}} w_{s \underline{j}}=\delta u_{s} v_{2 \underline{s} \bar{m}} x_{s(4 \ell+5)} \text {. }
$$




\section{The SIXTH SET OF SUMS}

The following formulas are also similar to (3.4)-(3.8) in [16]. Let $M_{0}=$ $W_{\bar{s}(\underline{\ell}+\underline{i})}-W_{\bar{s}(\ell+\bar{i})}, M_{1}=-W_{\bar{s}(\ell+\bar{i})}-W_{\bar{s}(\bar{\ell}+\bar{i})}, M_{2}=W_{\bar{s}(\bar{\ell}+\bar{i}+1)}-W_{\bar{s}(\bar{\ell}+\bar{i})}$ and $M_{3}=W_{\bar{s}(\bar{\ell}+\bar{i}+2)}+W_{\bar{s}(\bar{\ell}+\bar{i}+1)}$. For each $t=0,1,2,3$, the following identity holds.

$$
\frac{U_{\bar{s}}}{U_{\bar{s} \bar{k}}} \sum_{j=4 i+t}^{4 i+t+4 k+1}(-1)^{\frac{j(j+1)}{2}} W_{\bar{s} j}=M_{t} .
$$

The alternation according to $(-1)^{\frac{j(j-1)}{2}}$ gives similar identities. If the pairs $\{(+,-),(-,-),(+,-),(+,+)\}$ describe the signs in the four cases on the right hand side of $(7.1)$, then the pairs $\{(+,+),(+,-),(-,-),(+,-)\}$ give the signs in the formula for this alternation.

Let $M_{0}, M_{1}, M_{2}$ and $M_{3}$ be $W_{\bar{s}(\bar{\ell}+\bar{i})}-W_{\bar{s}(\bar{\ell}+i)}, W_{\bar{s}(\bar{\ell}+\bar{i}+1)}+W_{\bar{s}(\bar{\ell}+\bar{i})}$, $W_{\bar{s}(\bar{\ell}+\bar{i}+1)}-W_{\bar{s}(\bar{\ell}+\bar{i}+2)}$ and $-W_{\bar{s}(\bar{\ell}+\bar{i}+3)}-W_{\bar{s}(\bar{\ell}+\bar{i}+2)}$, respectively. For each $t=0,1,2,3$, the following identity holds.

$$
\frac{U_{\bar{s}}}{U_{\bar{s}(\bar{k}+1)}} \sum_{j=4 i+t}^{4 i+t+4 k+3}(-1)^{\frac{j(j+1)}{2}} W_{\bar{s} j}=M_{t} .
$$

The pairs $\{(-,-),(+,-),(+,+),(-,+)\}$ describe signs of terms on the right hand side for the alternation according to $(-1)^{\frac{j(j-1)}{2}}$ in $(7.2)$.

Let $M_{0}, M_{1}, M_{2}$ and $M_{3}$ be $\left[U_{\bar{s} \underline{k}}+U_{\bar{s} \bar{k}}\right] W_{\bar{s}(\underline{\ell}+\underline{i})},\left[U_{\bar{s} \underline{k}}-U_{\bar{s} \bar{k}}\right] W_{\bar{s}(\bar{\ell}+i)}$, $-\left[U_{\bar{s} \underline{k}}+U_{\bar{s} \bar{k}}\right] W_{\bar{s}(\bar{\ell}+\bar{i})}$ and $-\left[U_{\bar{s} \underline{k}}-U_{\bar{s} \bar{k}}\right] W_{\bar{s}(\bar{\ell}+\bar{i}+1)}$, respectively. For each $t=0,1,2,3$, the following identity holds.

$$
U_{\bar{s}} \sum_{j=4 i+t}^{4 i+t+4 k}(-1)^{\frac{j(j+1)}{2}} W_{\bar{s} j}=M_{t} .
$$

In the alternation according to $(-1)^{\frac{j(j+1)}{2}}$ above the signs of terms in the bracket are given by the pairs $\{(-,+),(+,+),(+,-),(-,-)\}$.

$$
\begin{aligned}
& \text { If } M_{0}=-\left[U_{\bar{s} \bar{k}}+U_{\bar{s}(\bar{k}+1)}\right] W_{\bar{s}(\bar{\ell}+\underline{i})}, M_{1}=-\left[U_{\bar{s} \bar{k}}-U_{\bar{s}(\bar{k}+1)}\right] W_{\bar{s}(\bar{\ell}+\bar{i})}, M_{2} \\
= & {\left[U_{\bar{s} \bar{k}}+U_{\bar{s}(\bar{k}+1)}\right] W_{\bar{s}(\bar{\ell}+\bar{i}+1)} \text { and } M_{3}=\left[U_{\bar{s} \bar{k}}-U_{\bar{s}(\bar{k}+1)}\right] W_{\bar{s}(\bar{\ell}+\bar{i}+2)} \text {, then } }
\end{aligned}
$$
for each $t=0,1,2,3$, the following identity holds.

$$
U_{\bar{s}} \sum_{j=4 i+t}^{4 i+t+4 k+2}(-1)^{\frac{j(j+1)}{2}} W_{\bar{s} j}=M_{t} .
$$


The pairs $\{(+,-),(-,-),(-,+),(+,+)\}$ describe signs of $U_{\bar{s} \bar{k}}$ and $U_{\bar{s}(\bar{k}+1)}$ in a similar formula for the alternation according to $(-1)^{\frac{j(j-1)}{2}}$.

When we replace the quotient $\frac{U_{\bar{s}}}{U_{\bar{s} \bar{k}}}$ with $\frac{V_{\underline{s}}}{V_{\underline{s}} \bar{k}}$ and every $\bar{s}$ with $\underline{s}$ in the rest of (7.1) we get the formula for the sum $\sum_{j=4 i+t}^{4 \underline{i}+t+4 k+1}(-1)^{\frac{j(j+1)}{2}} W_{\underline{s} j}$.

For the sum $\sum_{j=4 i+t}^{4 i+t+4 k+1}(-1)^{\frac{j(j-1)}{2}} W_{\underline{s} j}$, the paragraph after the identity (7.1) now applies.

Similarly, if we replace the quotient $\frac{U_{\bar{s}}}{U_{\bar{s}(\bar{k}+1)}}$ with $\frac{V_{\underline{s}}}{U_{\underline{s}}(\bar{k}+1)}$ and every $\bar{s}$ with $\underline{s}$ in the rest of (7.2) and on the right side every $W$ with $X$ we obtain the formula for the sum $\sum_{j=4 i+t}^{4 i+t+4 k+3}(-1)^{\frac{j(j+1)}{2}} W_{\underline{s} j}$.

For the sum $\sum_{j=4 i+t}^{4 i+t+4 k+3}(-1)^{\frac{j(j-1)}{2}} W_{\underline{s} j}$, the paragraph after the identity (7.2) now applies.

\section{The SEVENTH SET OF SUMS}

Some linear sums with binomial coefficients are also products. There are no sums of this type in [16]. However, there is a vast literature of papers that consider various sums in which binomial coefficients and (generalized) Fibonacci and/or Lucas numbers appear. Our references list only a few of these simply as examples of such papers.

Our first identity, for $i=0, a=0$ and $b=1$, is the Corollary 2 in a recent paper [10]. Hence, for $s=1, a=0, b=1$ and $p=1$ (when $W_{n}$ is the $n$-th Fibonacci number $F_{n}$ for every integer $n$ ), it agrees with the identity (3) in the more recent paper [7].

$$
\sum_{j=0}^{k}\left(\begin{array}{l}
k \\
j
\end{array}\right) W_{i+\underline{s} \underline{j}}=V_{\underline{s}}^{k} W_{i+\underline{s} k} .
$$

The following identity for $i=0, a=0$ and $b=1$, is Corollary 1 in [10]. Hence, for $s=0, a=0, b=1$ and $p=1$ it agrees with the identity (2.3) in [2].

$$
\frac{1}{\Delta^{\left\lfloor\frac{k}{2}\right\rfloor} U_{\bar{s}}^{k}} \sum_{j=0}^{k}\left(\begin{array}{l}
k \\
j
\end{array}\right) W_{i+\bar{s} \underline{j}}= \begin{cases}W_{i+\bar{s} k}, & \text { if } k \text { is even } \\
X_{i+\bar{s} k}, & \text { if } k \text { is odd. }\end{cases}
$$

The corresponding alternating sums are similar to (8.2) and (8.1).

$$
\frac{1}{\Delta^{\left\lfloor\frac{k}{2}\right\rfloor} U_{\underline{s}}^{k}} \sum_{j=0}^{k}(-1)^{j}\left(\begin{array}{l}
k \\
j
\end{array}\right) W_{i+\underline{s} \underline{j}}= \begin{cases}W_{i+\underline{s} k}, & \text { if } k \text { is even; } \\
-X_{i+\underline{s}} k, & \text { if } k \text { is odd. }\end{cases}
$$




$$
\sum_{j=0}^{k}(-1)^{j}\left(\begin{array}{l}
k \\
j
\end{array}\right) W_{i+\bar{s} \underline{j}}=(-1)^{k} V_{\bar{s}}^{k} W_{i+\bar{s} k} .
$$

The same form have the sums in which the binomial coefficient $\left(\begin{array}{l}k \\ j\end{array}\right)$ is multiplied with the integer $j$.

$$
\sum_{j=0}^{k} j\left(\begin{array}{l}
k \\
j
\end{array}\right) W_{i+\underline{s} \underline{j}}=k V_{\underline{s}}^{k-1} W_{i+\underline{s}(k+1)} .
$$

Let $M=k \Delta^{\left\lfloor\frac{k}{2}\right\rfloor-1} U_{\bar{s}}^{k-1}$.

$$
\sum_{j=0}^{k} j\left(\begin{array}{l}
k \\
j
\end{array}\right) W_{i+\bar{s} \underline{j}}= \begin{cases}M X_{i+\bar{s}(k+1),} & \text { if } k \text { is even; } \\
\Delta M W_{i+\bar{s}(k+1),} & \text { if } k \text { is odd. }\end{cases}
$$

Finally, the same holds for alternating sums of sums in (8.5) and (8.6). Let $M=k \Delta^{\left\lfloor\frac{k}{2}\right\rfloor-1} U_{\underline{s}}^{k-1}$.

$$
\begin{aligned}
& \sum_{j=0}^{k} j(-1)^{j}\left(\begin{array}{l}
k \\
j
\end{array}\right) W_{i+\underline{s} \underline{j}}= \begin{cases}M X_{i+\underline{s}}(k+1), & \text { if } k \text { is even; } \\
-M W_{i+\underline{s}}(k+1), & \text { if } k \text { is odd. }\end{cases} \\
& \sum_{j=0}^{k} j(-1)^{j}\left(\begin{array}{c}
k \\
j
\end{array}\right) W_{i+\bar{s} \underline{j}}=k(-1)^{k} V_{\bar{s}}^{k-1} W_{i+\bar{s}(k+1) .}
\end{aligned}
$$

\section{The EIGHTh SET OF SUMS}

The sums from the previous section for the numbers $w_{n}$ are a bit simpler.

$$
\sum_{j=0}^{k}\left(\begin{array}{l}
k \\
j
\end{array}\right) w_{i+\underline{s} j}=v_{s}^{k} w_{i+s k} .
$$

Let $A=(p+2)^{k}\left(u_{s+1}-u_{s}\right)^{\underline{k}}$.

$$
\frac{1}{A} \sum_{j=0}^{n}\left(\begin{array}{l}
n \\
j
\end{array}\right) w_{i+\bar{s} j}= \begin{cases}w_{i+\bar{s} k}, & \text { if } n=k \\
w_{i+\bar{s}(k+1)}+w_{i+\bar{s} k}, & \text { if } n=\bar{k} .\end{cases}
$$

When $s=0, i=0, a=0$ and $b=1$, the above identity appears as the identity (2.1) in [9].

When $i=0, a=0$ and $b=1$, the above identities (9.1) and (9.2) are similar to the identity (2.1) in [11].

$$
\frac{1}{\delta^{k} u_{s}^{n}} \sum_{j=0}^{n}(-1)^{j}\left(\frac{\underline{k}}{j}\right) w_{i+\underline{s} j}= \begin{cases}w_{i+\underline{s} k}, & \text { if } n=\underline{\underline{k}} \\ w_{i+\bar{k} s-1}-w_{i+\bar{k} s+1}, & \text { if } n=\bar{k}\end{cases}
$$


Let $A=(p-2)^{k}\left[u_{s+1}+u_{s}\right]^{n}$.

$$
\frac{1}{A} \sum_{j=0}^{n}(-1)^{j}\left(\begin{array}{l}
n \\
j
\end{array}\right) w_{i+\bar{s} j}= \begin{cases}w_{i+\bar{s} k}, & \text { if } n=\underline{k} \\
w_{\ell+\bar{s} k}-w_{\ell+\bar{s} k+1}, & \text { if } n=\bar{k}\end{cases}
$$

When $i=0, a=0$ and $b=1$, the above identities (9.3) and (9.4) are similar to the identity $(2.3)$ in [11].

$$
\sum_{j=0}^{k} j\left(\begin{array}{c}
k \\
j
\end{array}\right) w_{i+s j}=k v_{s}^{k-1} w_{i+s(k+1)}
$$

Let $A=n\left(u_{s+1}-u_{s}\right)^{n-1}$ and $M=(p+2)^{k-1}$.

$$
\frac{1}{A} \sum_{j=0}^{n} j\left(\begin{array}{l}
n \\
j
\end{array}\right) w_{i+\bar{s} j}= \begin{cases}M\left[w_{\ell+s \bar{k}+1}+w_{\ell+s} \bar{k}\right], & \text { if } n=\underline{k} \\
(p+2) M w_{\ell+\underline{s}(k+1)}, & \text { if } n=\bar{k} .\end{cases}
$$

When $s=0, i=0, a=0$ and $b=1$, the above identity appears as the identity (2.2) in [9].

When $i=0, a=0$ and $b=1$, the above identities (9.5) and (9.6) are similar to the identity $(2.2)$ in [11].

$$
\frac{1}{n u_{s}^{n-1}} \sum_{j=0}^{n} j(-1)^{j}\left(\begin{array}{l}
n \\
j
\end{array}\right) w_{i+\underline{s} j}= \begin{cases}\delta^{k-1} x_{i+s \bar{k}}, & \text { if } n=\underline{k} ; \\
-\delta^{k} w_{i+\underline{s}(k+1)}, & \text { if } n=\bar{k} .\end{cases}
$$

Let $A=n\left[u_{s+1}+u_{s}\right]^{n-1}, M=(p-2)^{k-1}$ and $N=w_{\ell+s \bar{k}+1}-w_{\ell+s \bar{k}}$.

$$
\frac{1}{A} \sum_{j=0}^{n} j(-1)^{j}\left(\begin{array}{l}
n \\
j
\end{array}\right) w_{i+\bar{s} j}= \begin{cases}M N, & \text { if } n=\underline{k} ; \\
(p-2) M w_{i+\bar{s}(k+1),}, & \text { if } n=\bar{k} ;\end{cases}
$$

When $i=0, a=0$ and $b=1$, the above identities (9.7) and (9.8) are similar to the identity (2.4) in [11].

\section{A sample PRoOF}

Each of our formulas can be discovered and proved with the help of a computer. Here we describe how to do this for the first formula in (2.1).

In Maple $\mathrm{V}$, the following code performs search for those sums of consecutive terms of the sequences $\left(W_{s j}\right)_{j \in \mathbb{Z}}$ which are products. In order to figure our some pattern, we consider the values $i, n$ and $s$ in some small ranges (for example, $1 \leq i \leq 4,1 \leq n \leq 10$ and $1 \leq s \leq 3$ ). We use the fact that the products have considerably smaller number of parts than the high order polynomials that do not factor.

for $i$ from 1 to 4 do for $n$ from 1 to 10 do for u from 1 to 3 do if nops (factor (add $(W(a, b, p,(2 * u+1) * j), j=i \ldots i+n)))<8$ then $\operatorname{print}([i, n, u]) ; f i ; o d ; o d ; o d ;$ 
Of course, the function $\mathrm{W}$ is the Binet form of the numbers $W_{n}$ from the introduction.

As a result of this search, we conclude that products show up only when the integers $n$ are either $4 k+1$ or $4 k+3$.

Next we repeat the above search for $n=4 k+1$ and for a small range of values $k$ take a closer look into the factors. One factor contains $a, b$ and $p$, while all other factors are polynomials only in $p$ and are (factors of the) polynomials $V_{n}$. The trick here is to multiply the sum $\sum_{j=i}^{i+n} W_{\bar{s} j}$ with $V_{\bar{s}}$ in order to conclude that these factors of the second kind (that do not contain $a$ and $b)$ are in fact $V_{\bar{s} \bar{k}}$.

Finally, the first factor (the one containing $a, b$ and $p$ ) does resemble numbers $W_{n}$ but not quite. The idea here is to select the index $n$ simply to eliminate the leading term of the first factor (considered as a polynomial in $p$ ). It is useful here to remember that polynomials (in $p$ ) on both sides of (2.1) have equal degrees. After we subtract this $W_{\bar{s} \mu}$ what remains is $W_{\bar{s} \lambda}$. The first factor is therefore $W_{\bar{s} \mu}+W_{\bar{s} \lambda}$.

Hence, so far we know that the identity

$$
(*) \quad V_{\bar{s}} \sum_{j=i}^{i+n} W_{\bar{s} j}=V_{\bar{s} \bar{k}}\left[W_{\bar{s} \lambda}+W_{\bar{s} \mu}\right]
$$

holds for some very small ranges of values $i, k$ and $s$. In order to prove that it is true for all values of $i, k$ and $s$, note that the left hand side of $(*)$ is $L H S=f \cdot(b-a \beta)-g \cdot(b-a \alpha)$, where

$$
f=f(\alpha, \beta)=\frac{\left(\alpha^{\bar{s}}+\beta^{\bar{s}}\right)\left(\alpha^{2 \bar{s} \bar{k}}-1\right) \alpha^{\bar{s} i}}{(\alpha-\beta)\left(\alpha^{\bar{s}}-1\right)}
$$

and $g=f(\beta, \alpha)$. The right hand side is $R H S=F \cdot(b-a \beta)-G \cdot(b-a \alpha)$, where

$$
F=F(\alpha, \beta)=\frac{\left(\alpha^{\bar{s} \bar{k}}+\beta^{\bar{s} \bar{k}}\right)\left(\alpha^{\bar{s}}+1\right) \alpha^{\bar{s}(\ell+k)}}{\alpha-\beta}
$$

and $G=F(\beta, \alpha)$. If we replace each $\beta$ in the difference $F-f$ with $-\frac{1}{\alpha}$ we obtain zero. Similarly, we conclude that $G=g$ so that $L H S=R H S$ and the first identity in (2.1) is true for all integer values of $i, k$ and $s$.

Remark 1. The method of proof for the identities in the seventh and the eighth group (the sums with binomial coefficients) is similar to the proofs of the Corollaries 1 and 2 in [10].

\section{Concluding Comments}

Each of our formulas for the sums of the numbers $W_{n}$ has a version for the corresponding sum of the numbers $X_{n}$. On the right hand sides the 
letters $W$ and $X$ are changed into the letters $X$ and $W$ and in the later case an extra factor $\Delta$ appears. Similarly, every formula for the sum of the dual numbers $w_{n}$ has a version for the corresponding sum of the dual numbers $x_{n}$. On the right hand sides the letters $w$ and $x$ are changed into the letters $x$ and $w$ and in the later case an extra factor $\delta$ appears.

The concluding comments in [16] explain the role of Russell's papers [17] and [18] in this area and how the contribution [16] and therefore also the present paper (only for the linear sums) and its sequel [5] (for the quadratic sums) want to explore those cases when these formulas are particularly simple. Of course, this is possible only for more specialized Horadam numbers like $W_{n}$ and $w_{n}$.

We hope that this paper for linear sums and its sequel [5] for quadratic sums constitute a partial realization of Melham's prediction "we expect that there is scope for further research along the lines that we set forth" on the first page of [16].

\section{REFERENCES}

[1] G. E. Bergum and V. E. Hoggatt, Jr., Sums and products for recurring sequences, Fibonacci Q.. 13 (2) (1975), 115-120.

[2] L. Carlitz and H. H. Ferns, Some Fibonacci and Lucas identities, Fibonacci Q., 8 (1) (1970), 61-73.

[3] Z. Čerin, On factors of sums of consecutive Fibonacci and Lucas numbers, Annales Mathematicae et Informaticae, 41 (2013), 19-25.

[4] Z. Čerin, Sums of products of generalized Fibonacci and Lucas numbers, Demonstr. Math., 42 (2) (2009), 247-258.

[5] Z. Cerin, Formulas for quadratic sums that involve generalized Fibonacci and Lucas numbers, Rad HAZU, Matematičke znanosti, (to appear).

[6] H. Freitag, On summations and expansions of Fibonacci numbers, Fibonacci Q., 11 (1) (1973), 63-71.

[7] M. Griffiths and A. Bramham, A combinatorial proof of a family of multinomialFibonacci identities, Integers, 14 (2014), \# A48.

[8] A. F. Horadam, Basic properties of a certain generalized sequence of numbers, Fibonacci Q., 3 (3) (1965), 161-176.

[9] M. A. Khan and H. Kwong, Some binomial identities associated with the generalized natural number sequence, Fibonacci Q., 49 (1) (2011), 57-65.

[10] E. Kiliç and N. Ömür, Some weighted sums of products of Lucas sequences, Integers, 13 (2013), \# A27.

[11] E. Kiliç, Y. Ulutaş and N. Ömür, Formulas for weighted binomial sums using the powers of terms of binary recurrences, Miskolc Math. Notes, 13 (1) (2012), pp. 5365.

[12] J. W. Layman, Certain general binomial-Fibonacci sums, Fibonacci Q., 15 (4) (1977), 362-366.

[13] C. T. Long, Some binomial Fibonacci identities, Applications of Fibonacci Numbers, 3: 241-254. Ed. G. E. Bergum et al. Dordrecht: Kluwer, 1990. 
[14] E. Lucas, Théorie des Fonctions Numériques Simplement Périodiques, Am. J. Math., 1 (1878), 184-240.

[15] R. S. Melham, Summation of reciprocals which involve products of terms from generalized Fibonacci sequences, Fibonacci Q., 38 (4) (2000), 294-298.

[16] R. S. Melham, Certain classes of finite sums that involve generalized Fibonacci and Lucas numbers, Fibonacci Q., 42 (1) (2004), 47-54.

[17] D. L. Russell, Summation of second-order recurrence terms and their squares, Fibonacci Q., 19 (4) (1981), 336-340.

[18] D. L. Russell, Notes on sums of products of generalized Fibonacci numbers, Fibonacci Q., 20 (2) (1982), 114-117.

[19] N. Sloane, On-Line Encyclopedia of Integer Sequences, http://www.research. att.com/ njas/sequences/.

[20] S. Vajda, Fibonacci $\&$ Lucas Numbers and the Golden Section: Theory and Applications, Ellis Horwood Limited, Chichester, England (John Wiley \& Sons, New York).

[21] C. R. Wall, Some congruences involving generalized Fibonacci numbers, Fibonacci Q., 17 (1) (1979), 29-33.

(Received: September 26, 2014)

Kopernikova 7

(Revised: December 16, 2014)

10020 Zagreb

Croatia

cerin@math.hr 